\title{
Analysis of Marketing Modes Based on Time-space Check-in Data
}

\author{
Haiping Wang ${ }^{1, *}$, Xiaolong Chen ${ }^{1}$ and Min Ren ${ }^{2}$ \\ ${ }^{1}$ School of Insurance, Shandong University of Finance and Economics No. 40, Shungeng Road, Jinan, Shandong province, China \\ ${ }^{2}$ School of Mathematics, Shandong University of Finance and Economics No. 40, Shungeng Road, Jinan, Shandong province, \\ China \\ ${ }^{*}$ Corresponding author
}

\begin{abstract}
We analyzed how to apply the check-in data to marketing areas by means of the current check-in software based on the theory of Time-Space Path. First, we explored possible marketing modes according to the path in the Time-Space theory and several kinds of interactive ways between people in timespace areas. Second, we chose the time-space coexistence and space coexistence conditions to make logical analysis. We found out the appropriate marketing time and locations by using the check-in data. Our contribution is to apply the time-space theory to the marketing analysis.
\end{abstract}

Keywords-time-space path; check-in; marketing modes

\section{INTRODUCTION}

Along with the popularization of mobile terminals such as mobile phones, laptops etc., all kinds of social network websites, e.g. Foursquare as the leader, have launched a new type of interactive mode, one key characteristic of which is to share one's own geographic information and actions with his or her social group - the name of this behavior is called "checkin". And "Qieke" is the name of Chinese translation according to its pronunciation. Based on this, a new concept appears- the Location-based Social network (LBS), which means the combination of Social Network Services (SNS) and Location, namely the Location-based Social Network Services.

Foursquare is such a kind of website which has made the record of 6 million on the registered users and owned 38.1576305 quadrillion check-in data in the year when it established. After the check-in concept was introduced to China, Foursquare even obtained the remarkably rapid development. According to the research consultant Eyrie, the number of users who enjoy the check-in service in China in 2013 is about 81 million [1]. The following Figure 1 shows this.

The emergence of check-in software and the rapid growth of users not only offer a large number of registered users' personal information for these similar websites, but also these websites can get a huge amount of location information about the users' mobile locations, which undoubtedly provides the extremely valuable social survey data. We attempt to explore how to make good use of these users' location information getting by the check-in software to carry out a more precise marketing.

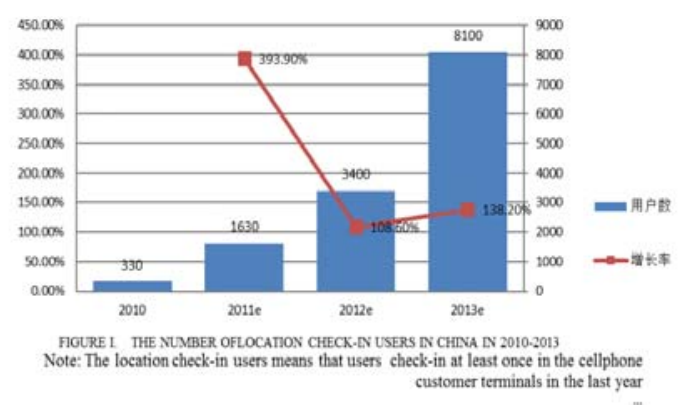

FIGURE I. THE NUMBER OF LOCATION CHECK IN USERS IN CHAINA IN 2010-2013

\section{LITERATURE REVIEW}

The related research on how to use the location information originates from the computer area. Some of them focus on the improvement of algorithm. They analyze the data of users' behavior trajectories to carry out trajectory simplifying (Chen Yukun, Jiang Kai and Zheng Yu, 2009 [5]; Chen Dianquan, 2011[6]). E.g. Yu and Shaw (2008) propose a kind of improved trajectory simplifying [3]. They dispose the walking and driving as different sections and consider the angle's change on adjacent tracing points. This makes the trajectory's simplification more reasonable.

Some research analyzes the POI (Points of Interest) matching (Kexin Xie, Ke Deng, Xiaofang Zhou, 2009[7]). Defu Lian, Xing Xie (2011) propose a location naming approach to provide concrete and meaningful location names to users based on their current location, time and check-in histories[11]. Min-Joong Lee and Chin-Wan Chung (2011) suggest an effective method to calculate the user similarity to capture the user's intention and interest using the semantics of the location [10]. Moreover, they calculate the similarity between different locations using the hierarchical location category.

Some research integrates the time data and space data to analyze users' habits according them. (Dianquan Chen, 2011)[6].Yang Ye, Yu Zheng, Yukun Chen, Jianhua Feng and Xing Xie (2011) proposes a novel notion of individual life pattern, that is, the life pattern normal form (the LP-normal form)[13]. They develop a LP-Mine framework to effectively retrieve life patterns from raw individual GPS data; describe which kind of life regularity can be discovered from location history and consider diverse properties to combine the 
significant places. Quannan Li etc. (2011) propose a hierarchical-graph-based similarity measurement (HGSM) framework by analyzing sequence property of people's movement behaviors and the hierarchy property of geographic spaces to model each individual's location history and to measure the similarity among users. These papers make good use of the computer technology and mathematics to process spatial-temporal data to infer user's behavior.

Other research builds a model of users' behavior characteristic in order to offer the individualized location service (Dianquan Chen and Zhaoyang, Huang, 2011[8]). By doing this, LBS operators could find out a lot of information from users' behavior data and offer coupon and discount service cooperated with the merchants. Lin Liao and Dieter Fox and Henry Kautz (2011) define a general framework for activity recognition by building upon and extending Relational Markov Networks[12]. Using the example of activity recognition from location data, we show that our model can represent a variety of features including temporal information such as time of day, spatial information extracted from geographic databases, and global constraints such as the number of homes or workplaces of a person.

\section{RELEVANT THEORETICAL BASIS}

Torsten Hägerstrand (1970), a famous geographer in Sweden, first introduces the concept of time geography in his research and defines it as a three dimensional Space - which is made up of a two-dimensional flat formed from the geographic locations coordinates where individuals taking activities and the third dimension which is composed of the time axis[2]. Everyone's activities' process in the time-space area can be described by using the Time-Space Path. The path for one person is composed of a set of vertical line segments and the diagonal lines. The vertical line segments mean a stopover in a certain place in the time period. The vertical height represents the staying time, and the diagonal lines mean the moving distance across a place to another place on its horizontal projection and the vertical height means the time spent by moving. Yu (2008) proposes four kinds of individual interactions on the basis of Torsten Hagerstrand's "time-space path" [2]. These are "Co-Existence", "Co-Location in Space"," Co-Location in Time" and "Co-Location in Either Space or Time". In order to better describe these relations proposed by $\mathrm{Yu}$, charts are made as shown in Figure 2.

\section{A. Co-Existence}

As shown in Figure 2.1 (a), it denotes that some people in a certain period of time contact with each other in the same position. And the projection of the dotted line overlapping on the plane is the location of their contacting; the interval projected on the time axis is the time interval when contacting.

\section{B. Co-Location in Space}

As shown in figure 2.1 (b), it refers to the individuals staying in the same location in the different time period, the projection of these dotted lines overlapping on the plane is the location of contacting with each other. Because Yu explores the situation of two individuals, which means there is no overlap between them (if there is the overlap, it will belong to the coexistence of time - space). In this article, we extend to the situation of multiple individuals. It denotes that there is no common overlapping interval between multiple individuals. For example, in Figure 2 (b), although part I and part IV have some overlap, but there are no common overlap area between part I, part II, part III and part IV, so it belongs to this situation. That is, all individuals do not stay in the same location in a certain time at the same time.

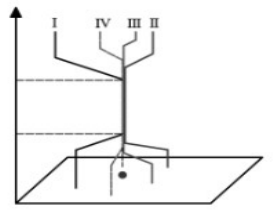

(a)

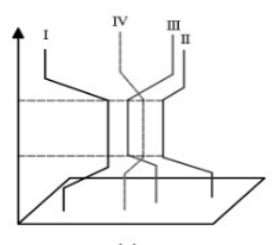

(c)

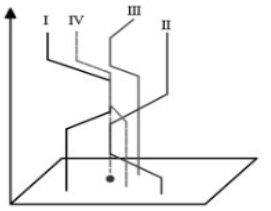

(b)

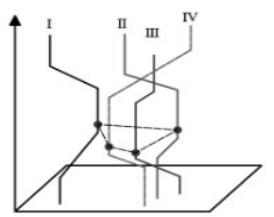

FIGURE II. THE FOUR FORMS ABOUT MULTIPLE INDIVIDUAL TIMESPACE INTERACTIONS (YU, 2008)

\section{Co-Location in Time}

As shown in figure 2 (c), it means that given an individual stays in different places in the same time but they can communicate and interact with each other by the remote electronic information tool. The projection interval on the time axis represented by the dotted lines is the time during which they communicate with one another. In reality, it mainly refers to the interactions between two or more individuals through telephone or Internet, such as call, video chat, remote meetings, etc. For multiple individuals who contact a certain information media at the same time, we argue that it can also be characterized in this kind of situation. For example, different individuals in different places watch the same TV program.

\section{Co-Location in Either Space or Time}

As shown in Figure 2 (d), which means that individuals in the different time and different place can communicate with each other by the time-lapse information tools. The most primitive way is traditional letters, but today, with the modern information technology's rapid development, it is clear that this kind of situation is becoming more common, such as email, voice message by the timely chat software, "offline file" transfer, phone messages, etc. in Figure2 (d), the spots in each line in the part I, II, III, and IV is the time and the place they stay in to accept information or send information mutually. Their projection in the plane and the time axis do not overlap with each other. 


\section{The APPlication OF THE TIME-SPACE PATH THEORY TO MARKETING}

\section{A. Co-Existence}

Each individual will concentrate in a specific location in a specific time period, which can be roughly divided into two situations in marketing, one is passive marketing, namely, short-term and small-scale marketing in a certain time interval and in a specific location. According to the data collected, the sellers can determine which specific time period and which specific locations are the population concentrated area, thus they can market their own products to the population; also by means of other centralized organization made up of specific populations, they can market their products, such as selling souvenirs around the World Cup stadiums. Another is the active marketing, which means that the population with special demand characteristics are concentrated together in a given period of time in order to carry out promotion such as product release conference and so on.

\section{B. Co-Location in Space}

Compared to time, this type of marketing should focus more on how to select locations. Because such group of people appear mostly in the different time periods and in the same location, such as railway stations and other places with large flow of people. So the choice of location is clearly more important. For this group of people, it is obviously more appropriate for the fixed billboards to be placed in the specific locations, such as bus station advertisement, highway billboards and the like.

\section{Co-Location in Time}

For this kind of group of people, it is more difficult to find them by the geographic location awareness. Because they interact with each other in the same time but in the different locations. Apparently when marketing to this population, marketers need to be more concerned about the time of selection. Because such group of people will focus on a specific media at a particular time, marketers should pay close attention to choose the time interval when relatively dense population concentrate and the marketing media which is focused on by more people. For example, the advertising price in CCTV golden schedule is relatively high.

\section{Co-Location in Either Space or Time}

This kind of marketing is a little difficult due to a high degree of inconsistency in the time and location. So generally it is carried out through a network media, such as an advertising marketing conducted on a particular webpage.

\section{THE MARKETING APPLICATIONS OF CHECK-IN DATA}

The check-in data is generally a set of three-dimensional data points. It is composed of the check-in coordinate (X, Y) and time (t) of every checkers. So we can only get information about time-space coexistence and space coexistence information through the data. But time coexistence and CoLocation in Either Space or Time data cannot be learned through the check-in data, so we mainly analyze the first two situations.

\section{A. Co-Existence}

The selection of a marketing location needs to take into account the time and place simultaneously. But due to the slight differences in location where a single individual lies (for example, it is difficult for two people's check-in place to achieve a perfect overlap). The check-in place must be clustered according to the location. Therefore, we present two ways in the paper.

Classified by grids: Classified by grids. That means assigning the check-in data's coordinate $(X, Y)$ to the corresponding grid, while adopting the form of threedimensional grid, the timeline is also divided into respective intervals. And thereby forming a facade of time and space similar to a magic cube, in which you can mark the threedimensional data acquired, and finally select choose the small cubes with higher frequency as a marketing focus. That is, you can carry on marketing within the space interval and time interval.

Classified by the respective functions: According to the functions of the scenery spots, for example, the website Foursquare divides them into eight categories. Namely, art entertainment, shops, food, nightlife, travel, education, parks outdoor and building. Literature [3] designs a method to calculate the prevalent degree, which can better judge and distinguish the class attribute of the node. And further, we can divide time into equidistant time intervals so as to arrive at the number of groups' occurrences in the appropriate time in each location node. Thus further analysis can be done.

\section{B. Co-Location in Space}

As mentioned earlier, the Co-Location in space is more concerned about the location and not the time. And for the people in this category, we need to analyze more on their movement paths. Because the billboards etc., can be contacted only if individuals coming through the place where they are. In analyzing these issues, we can resolve them mainly from the plane coordinates. In accordance with the foregoing ideas, the places on the plane are divided into equidistant intervals, and further each nodes are connected. Thereby their probable movement paths are formed. Each point which constitutes the entire movement paths is the location that individuals pass through. By calculating the length of the line within the range of individual paths in each plane, we can describe better the number of occurrences of each individual. Then the locations where the crowd appear more can be a better choice for marketing.

\section{ACKNOWLEDGMENT}

This research has been funded by National Natural Science Foundation of China (NSFC) Grant Research Project No.71172046, MOE (Ministry of Education in China) Youth Fund Project of Humanities and Social Sciences No.13YJC630162. 


\section{REFERENCES}

[1] 2010-2011 location check-in report, Iresearch Group, http://www.iresearch.com.cn/report/reportlist.aspx?page=16\&isfree=1

[2] T. Hagerstrand "What about People in Regional Science?" Papers and Proceedings of the Regional Science Association . 1970. Hägerstraand, Torsten. "What about people in regional science?." Papers in regional science 24.1 (1970): 7-24.

[3] H. Yu, , S-L Shaw,.., "Exploring potential human activities in physical and virtual spaces: a spatio-temporal GIS approach,” International Journal of Geographical Information Science, vol. 22, Issue 4, 2008, pp. 409-430.

[4] Lu Xin, Wang Chang-Hu, Yang Jiang-Ming, Pang Yanwei and Zhang Lei, "Photo2Trip: Generating travel routes from geo-tagged photos for trip planning, Proceedings of the ACM International Conference on Multimedia, Firenze, Italy. 2010, pp.143-152.

[5] Chen Yukun, Jiang Kai, Zheng Yu, "Trajectory simplification method for location-based social networking services,” LBSN' 09 Proceedings of the 2009 International Workshop on Location-based Social Networks, ACM New York, NY, USA, 2009, pp. 33-40.

[6] Chen Dianquan, "Analysis of users' behavior characteristics based on trajectories,” GNSS World of China, 2011, pp. 56-61.

[7] Xie Kexin, Deng Ke, Zhou Xiaofang. "From trajectories to activities: a spatio- temporal join approach,” LBSN' 09 Proceedings of the 2009 International Workshop on Location Based Social Networks, ACM New York, NY, USA, 2009, pp. 25-32.

[8] Chen Dianquan, Huang Zhaoyang, "Research on LBSN based on the location and its industrialization," Proceedings of the 2nd Chinese satellite navigation academic annual conference. Shanghai, 2011, pp. 144-147.

[9] Andrey Tietbohl Palma; Vania Bogorny; Bart Kuijpers; Luis Otavio Alvares, "A Clustering based approach for discovering interesting places in trajectories," Proceedings of the 2008 ACM symposium on applied computing. ACM New York, 2008, pp. 863-868.

[10] Lee Min-Joong and Chung Chin-Wan, “A user similarity calculation based on the location for social network services,” DASFAA 2011, Part I, LNCS 6587, 2011, pp. 38-52.

[11] Lian Defu, Xie Xing. "Learning location naming from user check-in histories," Proceedings of the $19^{\text {th }}$ ACM SIGSPATIAL International Conference on Advances in Geographic Informaiton Systems, ACM New York, 2011, pp.112-121.

[12] Liao Lin and Fox Dieter and Kautz Henry. "Location-based activity recognition using relational Markov networks," Proceedings of the $19^{\text {th }}$ international joint conference on Artificial intelligence, 2005, pp. 773778.

[13] Ye Yang, Zheng Yu, Chen Yukun, Feng Jianhua, Xie Xing. "Mining individual life pattern based on location history," Mobile Data Management - MDM , 2009: 1-10.

[14] Li Quannan, Zheng Yu, Xie Xing, Chen Yukun, Liu Wenyu, Wei-Ying Ma. "Mining user similarity based on location history," GIS '08 Proceedings of the 16th ACM SIGSPATIAL international conference on Advances in geographic information systems, Article No. 34, New York, USA, 008, pp.144-153. 